\title{
Supporting information for Photo-detection and Photoswitch based on Polarized Optical Response of Macroscopically Aligned Carbon Nanotubes
}

\author{
Ling Zhang ${ }^{1}$, Yang Wu ${ }^{1}$, Lei Deng ${ }^{2}$, Yi Zhoul, Changhong Liu ${ }^{1}$ and Shoushan Fan ${ }^{1}$ \\ ${ }^{1}$ Tsinghua-Foxconn Nanotechnology Research Center and Department of Physics, Tsinghua \\ University, Beijing 100084, People's Republic of China, and ${ }^{2}$ Department of Electrical Engineering, \\ Tsinghua University, Beijing 100084, People's Republic of China
}

Experimental Details

Scanning electron microscopy (SEM) analysis was performed under $10.0 \mathrm{kV}$ accelerating voltage in an FEI Sirion 200 microscope. The power values of both low power and high power modes for each laser are measured with a laser power meter and listed in Table S1.

The transmittance spectra of ACNTF in Figure 3A were recorded in a PerkinElmer Lambda 950 $\mathrm{UV} / \mathrm{vis}$ spectrometer. A depolarizer whose depolarization efficiency of greater than $98 \%$ is assembled in the system to ensure the same intensity along different directions of incident laser. A series of background light intensities are measured to eliminate the potential interference from inconsistent intensity along different directions. In Figure 3D, there is no original absorption data from included angle 325 to 30 degree, due to continuous rotation limitation of polarizer in the spectrometer.

Table S1. The low power and high power values at different laser wavelength in Figure 3C.

\begin{tabular}{lrrrr} 
& $405 \mathrm{~nm}$ & $450 \mathrm{~nm}$ & $650 \mathrm{~nm}$ & $973 \mathrm{~nm}$ \\
\hline Low Power & $200 \mathrm{~mW}$ & $250 \mathrm{~mW}$ & $150 \mathrm{~mW}$ & $350 \mathrm{~mW}$
\end{tabular}




High Power $\quad 400 \mathrm{~mW} \quad 500 \mathrm{~mW} \quad 250 \mathrm{~mW} \quad 800 \mathrm{~mW}$

21 The preparation of 'wavelength lock' in Figure 4 is presented below: the aligned CNT film

22 (ACNTF) sample was fixed on the stage with the heating end placed at the center of the stage.

23 During the rotation operation, the laser and polarizer were fixed and only the stage together with

24 ACNTF sample rotated. A thermocouple was connected to an Arduino processor to deliver the

25 thermoelectric potential (TP) data. An electromagnetic lock was connected to the output port of the

26 Arduino processor. When a series of TP data are inputted and recorded, the processor would judge

27 the incident laser wavelength by detecting the minimum and maximum values in the data series. If

28 the calculated wavelength is consistent with prestored criterion, the 'key' of the lock, an 'open door'

29 order will be sent from the processor to disable the electromagnetic lock.

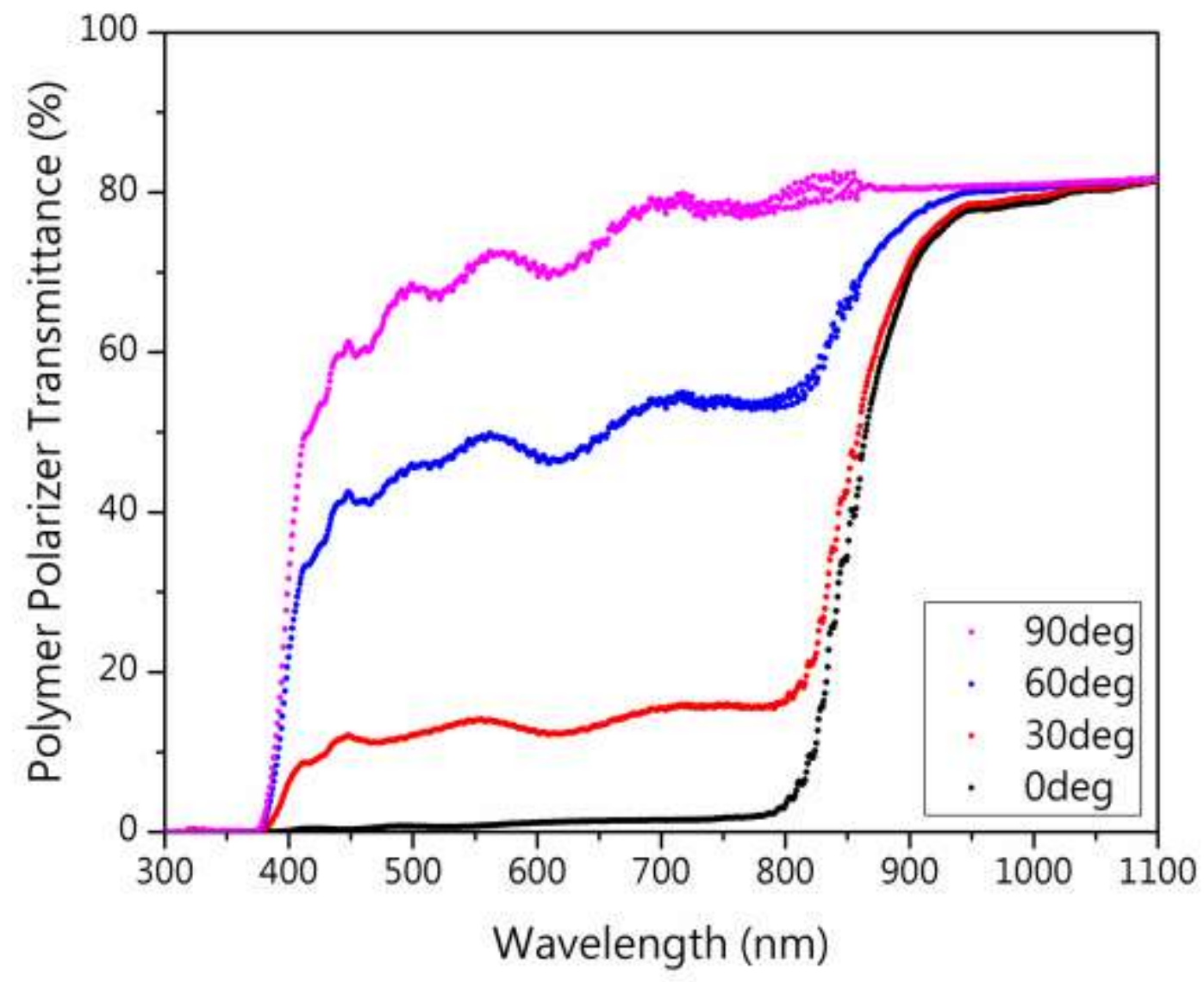


Figure S1. Transmittance spectra of polarized lights as a function of wavelength at selected included angles between the polarization of light and the polymer polarizing direction.

To further emphasize the monotony of transmittance of ACNTF, we measured the transmittance spectra of the commercial polymer polarizer. Due to film interference, the transmittance is not monotonous when the light wavelength changes, and only covers the range of 400-800 nm.

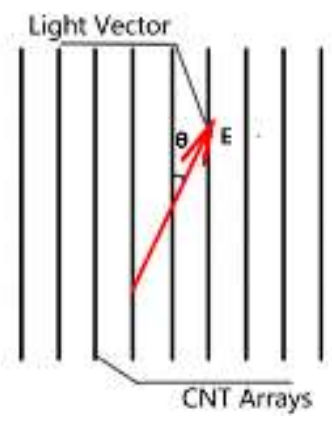

38 Figure S2. The model of light vector goes through the CNT arrays. The red arrow indicates the polarization direction of light vector, with an included angle $\theta$ with CNT alignment.

We founded a simple model to explain the experiments. Let $\theta$ be the included angle between polarized light vector $\mathrm{E}$ and alignment of CNT. We decomposite E along CNT alignment. The

42 light factor parallel to $\mathrm{CNT}$ is $\mathrm{E} \cos \theta$, and the light factor perpendicular to $\mathrm{CNT}$ is Esin $\theta$. Under a 43 specific wavelength $\lambda$, let $\alpha_{\lambda}$ be the CNT's absorption to light factor in parallel direction, and $\beta_{\lambda}$ 44 be the absorption in perpendicular direction. Therefore, $E_{\lambda}(\theta)$, the total optical absorption by CNT is 45 the composition of both parts: $E_{\lambda}(\theta)=\mathrm{E} \sqrt{\alpha_{\lambda}{ }^{2} \cos ^{2} \theta+\beta_{\lambda}{ }^{2} \sin ^{2} \theta}$. As the light intensity $\mathrm{I} \propto \mathrm{E}^{2}$, the 46 actual absorption

$$
\gamma_{\lambda}(\theta)=\frac{\mathrm{I}_{\lambda}(\theta)}{I}=\left(\frac{E_{\lambda}(\theta)}{E}\right)^{2}=\alpha_{\lambda}^{2} \cos ^{2} \theta+\beta_{\lambda}^{2} \sin ^{2} \theta
$$

48 We explain why $\gamma_{\lambda}^{-\frac{1}{2}}(\theta)$ has an ellipse form in polar coordinates with an aspect ratio of $\frac{\alpha_{\lambda}}{\beta_{\lambda}}$ to 49 describe the ellipse shape. In the Cartesian coordinates, the ellipse has the equation form of 
$50 \frac{x^{2}}{a^{2}}+\frac{y^{2}}{b^{2}}=1$. By using the relationship of $\mathrm{x}=\rho \cos \theta$ and $\mathrm{y}=\rho \sin \theta$, in which $\rho$ and $\theta$ are radius

51 and angle respectively, we can rewrite the ellipse equation as

52

$$
\frac{1}{\rho^{2}}=\frac{\cos ^{2} \theta}{a^{2}}+\frac{\sin ^{2} \theta}{b^{2}}
$$

53 We compare (1) and (2), and it is obvious that $\gamma_{\lambda}^{-\frac{1}{2}}(\theta)$ has an ellipse form, with aspect ratio of $\frac{\alpha_{\lambda}}{\beta_{\lambda}}$.

54 To derive $\frac{\alpha_{\lambda}}{\beta_{\lambda}}$, we denote $I_{\lambda}(\theta) \propto E_{\lambda}{ }^{2}(\theta) \propto U_{\lambda}{ }^{2}(\theta)$, in which $U_{\lambda}(\theta)$ is the TP generated by light

55 intensity $I_{\lambda}(\theta)$, if assuming sample a constant energy conversion efficiency. Therefore, TP has the

56 form of $U_{\lambda}(\theta)=C \mathrm{E} \sqrt{\alpha_{\lambda}{ }^{2} \cos ^{2} \theta+\beta_{\lambda}{ }^{2} \sin ^{2} \theta} . C$ is a constant. It can be easily derived that

$$
\frac{\alpha_{\lambda}}{\beta_{\lambda}}=\frac{U_{\lambda}(0 \mathrm{deg})}{U_{\lambda}(90 \mathrm{deg})}(3)
$$

58 Therefore, the ratio between the maximum and minimum of absorption corresponds to the aspect 59 ratio of ellipses.

60 